\title{
19. STABLE ISOTOPE STRATIGRAPHY AND PALEOCEANOGRAPHY OF SITES 502 AND 5031
}

\author{
L. D. Keigwin, Jr., ${ }^{2}$ Graduate School of Oceanography, University of Rhode Island, Kingston, Rhode Island
}

\begin{abstract}
Analyses of stable isotopes of monospecific planktonic foraminifers ( $G$. quadrilobatus group) and monogeneric benthic foraminifers (Cibicidoides spp.) from late Neogene Atlantic Site 502 and Pacific Site 503 were conducted in order to determine the paleoceanographic changes resulting from the late Neogene uplift of the Panama Isthmus and from climatic cooling.

In general, results at each site are similar to those from previous studies for the late Miocene and late Pliocene time interval, documenting the late Miocene (6 Ma) shift in carbon isotopes and the inferred growth of permanent Northern Hemisphere continental ice sheets beginning about 3.2 Ma. Comparison of Atlantic-Pacific planktonic-benthic isotope data for four stratigraphic intervals $(\sim 6-8, \sim 5-6, \sim 3-5$, and $\sim 2-3 \mathrm{Ma})$ suggests that increasing isolation of Atlantic and Pacific low-latitude waters may be related to the emergence of the Panama Isthmus. The contrast between Atlantic and Pacific benthic foraminiferal $\delta^{13} \mathrm{C}$ increased in two steps from $0.60 \%$ to $1 \%$ (the modern contrast) at about $6 \mathrm{Ma}$ and $3 \mathrm{Ma}$. The first increase $(0.15 \%$ ) may represent the end of previously limited deep-water communication between the Atlantic and Pacific at the present location of Panama. The second increase $(0.25 \%)$ may be due to increased production of North Atlantic Deep Water. This probably reflects the development of modern deep-sea circulation.

The $\delta^{18} \mathrm{O}$ of planktonic foraminifers begins to increase in Atlantic Site 502 at $4.2 \mathrm{Ma}$ and may reflect the increasing salinity of the North Atlantic Ocean arising from diminishing surface-water exchange across Panama. This increase is clearly shown by contrasting the $\delta^{18} \mathrm{O}$ of Atlantic and Pacific planktonic foraminifers, as well as the $\delta^{18} \mathrm{O}$ of planktonic and benthic foraminifers at Site 502. This inferred increase in surface-water salinity begins at the time of increasing provinciality of Atlantic and Pacific planktonic foraminifers.
\end{abstract}

\section{INTRODUCTION}

Deep Sea Drilling Project (DSDP) Sites 502 and 503, which were hydraulically piston cored, provide a unique opportunity to compare the histories of the westernmost North Atlantic and the eastern equatorial Pacific oceans. Each site has an apparently continuous sedimentary sequence of about $8 \mathrm{~m}$.y. duration from near the same water depth. In addition, each is located near the Panama Isthmus (Table 1) and thus records the effects of the late Neogene transition from an open Atlantic- $\mathrm{Pa}-$ cific connection to complete separation of the tropical oceans (Keigwin, this volume, and references cited therein). The recovery of sediment from Site 502 extends the pelagic record of the Colombian Basin back three to four million years past that recovered at nearby Site 154A (Edgar et al., 1973). The section recovered at Site 503 is more suitable for high resolution studies than that from nearby Site 83, which was only spot-cored and was disturbed by the rotary drilling process (Hays et al., 1972). The effects of the growing Atlantic-Pacific isolation on the planktonic foraminiferal fauna from Sites 502 and 503 are discussed in another chapter (Keigwin, this volume), while the results of analyzing the stable isotopes of planktonic and benthic foraminifers are discussed in this chapter.

\section{METHODS}

Analyses of stable isotopes were performed following standard procedures (Keigwin, 1979) on foraminifers selected from the same

\footnotetext{
${ }^{1}$ Prell, W. L., Gardner, J. V., et al., Init. Repts. DSDP, 68: Washington (U.S. Govt. Printing Office).

2 Present address: Department of Geology and Geophysics, Woods Hole Oceanographic Institution, Woods Hole, Massachusetts.
}

Table 1. Site locations.

\begin{tabular}{ccccc}
\hline Site & Latitude & Longitude & Depth (m) & Geographic Feature \\
\hline 502 & $1^{\circ} 29.4^{\prime} \mathrm{N}$ & $79^{\circ} 22.7^{\prime} \mathrm{W}$ & 3051 & Ridge in West Colombian Basin \\
503 & $4^{\circ} 04.04^{\prime} \mathrm{N}$ & $95^{\circ} 38.21^{\prime} \mathrm{W}$ & 3672 & North flank of Galapagos Ridge \\
\hline
\end{tabular}

samples used for semiquantitative biostratigraphy (Keigwin, this volume). Results are presented in Table 2 and Figure 1 and 2 in delta notation $(\delta)$ with respect to the standard PDB following the calibration of Keigwin (1980).

Various workers (Duplessy et al., 1980; Woodruff et al., 1981; Belanger et al., 1981; Graham et al., in prep.) have indicated that species of the benthic foraminifer Cibicidoides give reliable $\delta^{18} \mathrm{O}$ and $\delta^{13} \mathrm{C}$ results. No single species of Cibicidoides was abundant in all samples from both Leg 68 sites, so Cibicidoides wuellerstorfi was analyzed from Site 502 and $C$. kullenbergi was selected at Site 503. All benthic foraminifers were from the size fraction greater than $175 \mu \mathrm{m}$. In some samples where both species were present in sufficient abundance, paired analyses were performed. The results of 15 such pairs (Table 3 ) show that isotope differences between these species are within the precision of analysis. For the following discussion I assume that these differences are insignificant and that these two species may be used interchangeably within cores of a site and between sites. Variability among species of this genus has also been found to be within analytical precision by Duplessy et al. (1980) and Woodruff et al. (1981). At both Sites 502 and 503 members of the planktonic foraminiferal group Globigerinoides trilobus-quadrilobatus-sacculifera were chosen for analysis from the size fraction 175 to $295 \mu \mathrm{m}$.

Initially, the benthic foraminifer Oridorsalis tener was chosen for isotope analysis at both Sites 502 and 503 . However, Belanger et al. (1981) have shown that Oridorsalis neither precipitates its calcite in isotopic equilibrium with the $\delta^{13} \mathrm{C}$ of total $\mathrm{CO}_{2}$ in seawater, nor follows apparent oxygen utilization (AOU) in a consistent manner as does the $\delta^{13} \mathrm{C}$ of total $\mathrm{CO}_{2}$. Hence, analysis of $O$. tener was discontinued after the analyses presented in Table 2 had been performed.

To simplify comparing results at the two sites, I have divided each record into four time intervals within which the mean and standard deviation are calculated (Table 4). Datum levels marking each interval are: a change in carbon isotopes around $6 \mathrm{Ma}$, the Miocene/Pliocene boundary ( $5.26 \mathrm{Ma}$, based on the first occurrence of G. tumida), and 
Table 2. Leg 68 stable isotope results.

\begin{tabular}{|c|c|c|c|c|c|c|c|c|c|}
\hline \multirow{2}{*}{$\begin{array}{c}\text { Sample } \\
\text { (level in } \mathrm{cm} \text { ) }\end{array}$} & \multirow{2}{*}{$\begin{array}{l}\text { Depth } \\
\text { (m) }\end{array}$} & \multicolumn{2}{|c|}{$\underset{\text { wuellerstorfi }}{C .}$} & \multicolumn{2}{|c|}{$\begin{array}{c}C . \\
\text { kullenbergi }\end{array}$} & \multicolumn{2}{|c|}{$\begin{array}{c}G . \\
\text { quadrilobatus }\end{array}$} & \multicolumn{2}{|c|}{$\begin{array}{c}0 . \\
\text { tener }\end{array}$} \\
\hline & & ${ }^{18} \mathrm{O}$ & ${ }^{813} \mathrm{C}$ & ${ }^{18} \mathrm{O}$ & ${ }_{8}^{13} \mathrm{C}$ & ${ }^{18} \mathrm{O}$ & ${ }_{8}^{13} \mathrm{C}$ & ${ }^{18} \mathrm{O}$ & ${ }^{\delta^{13} \mathrm{C}}$ \\
\hline \multicolumn{10}{|l|}{ 502B } \\
\hline $\begin{array}{l}1-1,000 \\
11, \mathrm{CC} \\
12, \mathrm{CC} \\
13, \mathrm{CC} \\
14, \mathrm{CC} \\
15, \mathrm{CC} \\
16, \mathrm{CC} \\
17, \mathrm{CC} \\
18, \mathrm{CC}\end{array}$ & $\begin{array}{r}0.00 \\
45.69 \\
48.92 \\
54.41 \\
58.53 \\
60.46 \\
64.38 \\
71.90 \\
76.41\end{array}$ & $\begin{array}{l}1.90 \\
2.11 \\
2.41 \\
2.94 \\
1.92 \\
2.08 \\
1.59 \\
1.77 \\
2.08\end{array}$ & $\begin{array}{l}0.86 \\
0.45 \\
0.45 \\
0.69 \\
0.56 \\
0.52 \\
0.40 \\
0.60 \\
0.48\end{array}$ & & & $\begin{array}{l}-1.82 \\
-1.37 \\
-1.10 \\
-0.76 \\
-1.25 \\
-1.08 \\
-1.00 \\
-0.98 \\
-1.56\end{array}$ & $\begin{array}{l}1.10 \\
0.88 \\
0.79 \\
0.65 \\
0.95 \\
1.28 \\
1.25 \\
0.92 \\
0.82\end{array}$ & & \\
\hline \multicolumn{10}{|l|}{$502 A$} \\
\hline $\begin{array}{l}19, \mathrm{CC} \\
20, \mathrm{CC} \\
21, \mathrm{CC} \\
22, \mathrm{CC} \\
23, \mathrm{CC} \\
24, \mathrm{CC} \\
25, \mathrm{CC} \\
26, \mathrm{CC} \\
27, \mathrm{CC} \\
28, \mathrm{CC} \\
29, \mathrm{CC} \\
30, \mathrm{CC} \\
32, \mathrm{CC} \\
33, \mathrm{CC} \\
34, \mathrm{CC} \\
35, \mathrm{CC} \\
36, \mathrm{CC} \\
37, \mathrm{CC}\end{array}$ & $\begin{array}{r}80.45 \\
85.46 \\
89.56 \\
94.27 \\
98.78 \\
103.18 \\
107.27 \\
111.85 \\
116.12 \\
119.34 \\
122.83 \\
125.92 \\
131.62 \\
134.46 \\
137.30 \\
140.77 \\
143.09 \\
145.14\end{array}$ & $\begin{array}{l}2.11 \\
2.37 \\
1.56 \\
1.59 \\
1.67 \\
1.78 \\
1.58 \\
1.57 \\
1.74 \\
1.60 \\
1.52 \\
1.61 \\
1.54 \\
1.71 \\
1.64 \\
1.46 \\
1.34 \\
1.62\end{array}$ & $\begin{array}{l}0.46 \\
0.65 \\
0.60 \\
0.65 \\
0.49 \\
0.49 \\
0.42 \\
0.18 \\
0.44 \\
0.09 \\
0.18 \\
0.41 \\
0.33 \\
0.36 \\
0.50 \\
0.09 \\
0.28 \\
0.48\end{array}$ & & & $\begin{array}{l}-0.94 \\
-1.30 \\
-1.29 \\
-1.57 \\
-1.51 \\
-1.59 \\
-1.43 \\
-1.84 \\
-1.68 \\
-1.98 \\
-1.89 \\
-1.86 \\
-1.97 \\
-1.92 \\
-1.77 \\
-2.03 \\
-2.31 \\
-1.75\end{array}$ & $\begin{array}{l}1.17 \\
1.13 \\
1.58 \\
0.71 \\
1.15 \\
0.83 \\
0.90 \\
0.72 \\
0.92 \\
0.62 \\
1.01 \\
0.86 \\
0.74 \\
0.92 \\
0.88 \\
0.71 \\
1.02 \\
1.29\end{array}$ & & \\
\hline $38, \mathrm{CC}$ & 147.49 & 1.58 & 0.74 & & & & & & \\
\hline $\begin{array}{l}39, \mathrm{CC} \\
40, \mathrm{CC}\end{array}$ & $\begin{array}{l}149.86 \\
152.85\end{array}$ & $\begin{array}{l}1.95 \\
1.74\end{array}$ & $\begin{array}{l}0.43 \\
0.60\end{array}$ & & & $\begin{array}{l}-1.71 \\
-2.10\end{array}$ & $\begin{array}{l}1.36 \\
1.13\end{array}$ & & \\
\hline $41, \mathrm{CC}$ & 155.51 & 1.35 & 0.11 & & & -1.66 & 1.23 & & \\
\hline $42, \mathrm{CC}$ & 158.45 & 1.49 & 0.52 & & & -2.08 & 0.98 & & \\
\hline $43, \mathrm{CC}$ & 161.00 & 1.79 & 0.61 & & & -2.24 & 1.35 & & \\
\hline $44, \mathrm{CC}$ & 163.48 & 1.28 & 0.77 & & & -2.24 & 1.66 & & \\
\hline $45, \mathrm{CC}$ & 166.04 & 1.51 & 0.66 & & & -2.17 & 1.46 & & \\
\hline $46, \mathrm{CC}$ & 168.15 & 1.63 & 0.76 & & & -2.34 & 1.82 & & \\
\hline $47, \mathrm{CC}$ & 171.24 & 1.44 & 0.36 & & & -2.40 & 1.66 & & \\
\hline $\begin{array}{l}48, C C \\
50, C C\end{array}$ & $\begin{array}{l}172.98 \\
177.63\end{array}$ & $\begin{array}{l}1.54 \\
1.40\end{array}$ & $\begin{array}{l}0.75 \\
0.46\end{array}$ & & & $\begin{array}{l}-2.13 \\
-2.31\end{array}$ & $\begin{array}{l}1.86 \\
1.47\end{array}$ & & \\
\hline $51, \mathrm{CC}$ & 180.36 & 1.54 & 0.50 & & & -2.23 & 1.05 & 2.25 & -1.40 \\
\hline $52, \mathrm{CC}$ & 182.33 & 1.34 & 0.51 & & & -1.97 & 1.42 & 2.43 & -1.37 \\
\hline $53, \mathrm{CC}$ & 183.94 & 1.76 & 0.54 & & & -2.21 & 1.16 & 2.39 & -1.42 \\
\hline $54, \mathrm{CC}$ & 187.03 & 1.64 & 0.30 & & & $\begin{array}{l}-1.70 \\
-1.80\end{array}$ & $\begin{array}{l}1.32 \\
1.36\end{array}$ & 2.19 & -1.35 \\
\hline $55, \mathrm{CC}$. & 189.15 & 1.71 & 0.32 & & & -2.24 & 0.89 & 2.21 & -1.38 \\
\hline $56, \mathrm{CC}$ & 191.06 & 1.54 & 0.24 & & & -2.11 & 1.39 & 2.03 & -1.63 \\
\hline $57, \mathrm{CC}$ & 193.06 & 1.73 & 0.26 & & & -2.17 & 1.23 & 2.19 & -1.43 \\
\hline $58, \mathrm{CC}$ & 195.02 & 1.25 & 0.60 & & & -2.08 & 1.30 & & \\
\hline $59, \mathrm{CC}$ & 195.64 & 1.71 & 0.41 & & & -2.12 & 1.49 & & \\
\hline $60, \mathrm{CC}$ & 198.97 & 1.47 & 0.81 & & & -2.52 & 2.18 & & \\
\hline $61, \mathrm{CC}$ & 200.30 & 1.55 & 0.93 & & & -2.04 & 1.92 & 2.22 & -0.61 \\
\hline $62, \mathrm{CC}$ & 201.68 & 1.49 & 0.97 & & & -1.86 & 1.69 & 2.12 & -0.47 \\
\hline $63, \mathrm{CC}$ & 204.57 & 1.75 & 1.26 & & & -2.18 & 2.01 & 2.33 & -0.04 \\
\hline $65, \mathrm{CC}$ & 209.34 & 1.87 & 0.69 & & & -1.75 & 1.43 & 2.19 & -0.49 \\
\hline $66, \mathrm{CC}$ & 210.86 & 1.86 & 0.68 & & & -1.56 & 1.66 & 2.33 & -0.37 \\
\hline $67, \mathrm{CC}$ & 211.93 & 1.72 & 0.90 & 1.60 & 0.75 & -2.24 & 1.97 & 2.06 & -0.60 \\
\hline $68, \mathrm{CC}$ & 214.88 & 1.68 & 0.93 & & & -1.63 & 1.72 & & \\
\hline \multicolumn{10}{|l|}{$502 C$} \\
\hline $28, \mathrm{CC}$ & 213.08 & 1.84 & 0.88 & & & -2.46 & 2.24 & & \\
\hline $29, \mathrm{CC}$ & 215.22 & $\begin{array}{l}1.82 \\
1.65\end{array}$ & $\begin{array}{l}0.45 \\
0.37\end{array}$ & 1.65 & 0.45 & -1.91 & 1.54 & & \\
\hline $30, \mathrm{CC}$ & 216.19 & 1.74 & 0.73 & 1.79 & 0.75 & -1.88 & 1.80 & & \\
\hline $31, \mathrm{CC}$ & 218.75 & 2.04 & 0.82 & 1.75 & 0.56 & -2.05 & 1.38 & & \\
\hline $32, \mathrm{CC}$ & 221.18 & 1.75 & 0.92 & 1.81 & 0.74 & -1.91 & 1.68 & & \\
\hline $33, \mathrm{CC}$ & 223.88 & 1.74 & 0.85 & & & -1.84 & 1.93 & & \\
\hline $34, \mathrm{CC}$ & 225.26 & 1.74 & 0.84 & 1.73 & 0.81 & -1.51 & 1.48 & & \\
\hline $35, C C$ & 226.28 & 1.68 & 0.91 & 1.57 & 0.95 & -1.89 & 1.62 & & \\
\hline $36, \mathrm{CC}$ & 227.00 & 1.53 & 0.88 & 1.76 & 0.73 & -2.02 & 1.93 & & \\
\hline $37, \mathrm{CC}$ & 227.81 & 1.86 & 0.92 & 1.83 & 0.67 & -2.04 & 1.71 & & \\
\hline $503 \mathrm{~A}$ & & & & & & & & & \\
\hline $1-1,000$ & 0.00 & 2.47 & -0.12 & & & & & & \\
\hline \multicolumn{10}{|l|}{ 503B } \\
\hline $8, \mathrm{CC}$ & 33.52 & 2.39 & -0.48 & 2.17 & -0.31 & -1.74 & 0.97 & & \\
\hline $10, \mathrm{CC}$ & 41.97 & $\begin{array}{l}2.23 \\
2.16\end{array}$ & $\begin{array}{l}-0.41 \\
-0.29\end{array}$ & 2.23 & -0.42 & -1.60 & 1.17 & & \\
\hline $11, \mathrm{CC}$ & 46.57 & 2.70 & -0.93 & & & -1.38 & 0.56 & & \\
\hline & 50.72 & 2.51 & -0.65 & & & $\begin{array}{l}-1.53 \\
-1.65\end{array}$ & $\begin{array}{l}0.89 \\
0.91\end{array}$ & & \\
\hline $13, \mathrm{CC}$ & 54.70 & 1.92 & -0.32 & & & $\begin{array}{l}-2.14 \\
-2.19\end{array}$ & $\begin{array}{l}1.36 \\
1.56\end{array}$ & & \\
\hline $14, \mathrm{CC}$ & 59.12 & $\begin{array}{l}2.28 \\
2.19\end{array}$ & $\begin{array}{l}-0.71 \\
-0.55\end{array}$ & & & -1.92 & 1.32 & & \\
\hline $15, \mathrm{CC}$ & 63.80 & 1.98 & -0.03 & & & -2.14 & 1.49 & & \\
\hline
\end{tabular}

Table 2. (Continued).

\begin{tabular}{|c|c|c|c|c|c|c|c|c|c|}
\hline \multirow{2}{*}{$\begin{array}{c}\text { Sample } \\
\text { (level in cm) }\end{array}$} & \multirow{2}{*}{$\begin{array}{l}\text { Depth } \\
(\mathrm{m})\end{array}$} & \multicolumn{2}{|c|}{$\begin{array}{c}C . \\
\text { wuellerstorfi }\end{array}$} & \multicolumn{2}{|c|}{$\begin{array}{c}C . \\
\text { kullenbergi }\end{array}$} & \multicolumn{2}{|c|}{$\begin{array}{c}G . \\
\text { quadrilobatus }\end{array}$} & \multicolumn{2}{|c|}{$\begin{array}{c}0 . \\
\text { tener }\end{array}$} \\
\hline & & ${ }_{8}^{180}$ & ${ }_{{ }^{13}}^{13} \mathrm{C}$ & 8180 & ${ }^{13} \mathrm{C}$ & ${ }_{818}^{18} \mathrm{O}$ & ${ }_{8}^{13} \mathrm{C}$ & ${ }_{8}^{18} \mathrm{O}$ & ${ }_{\delta^{13} \mathrm{C}}$ \\
\hline \multicolumn{10}{|l|}{ 503B (Cont.) } \\
\hline $\begin{array}{l}16-1,106 \\
16-2,106\end{array}$ & $\begin{array}{l}64.90 \\
66.80\end{array}$ & & & $\begin{array}{l}2.51 \\
1.96\end{array}$ & $\begin{array}{l}-0.76 \\
-0.47\end{array}$ & & & & \\
\hline $16, C C$ & 68.36 & 2.14 & -0.43 & & & -1.91 & 1.09 & & \\
\hline $\begin{array}{l}17-1,106 \\
17-2,106\end{array}$ & $\begin{array}{l}69.76 \\
71.27\end{array}$ & & & $\begin{array}{l}1.82 \\
1.99\end{array}$ & $\begin{array}{l}-0.26 \\
-0.56\end{array}$ & & & & \\
\hline $17, \mathrm{CC}$ & 72.49 & 1.78 & -0.09 & & & -2.03 & 1.36 & & \\
\hline $18, \mathrm{CC}$ & 77.61 & 1.77 & -0.58 & 1.76 & -0.56 & -2.14 & & & \\
\hline $19, \mathrm{CC}$ & 80.96 & 1.98 & -0.20 & 1.80 & -0.37 & -2.51 & 1.46 & & \\
\hline $21, \mathrm{CC}$ & 90.81 & 1.80 & -0.24 & 1.84 & -0.38 & -2.34 & 1.32 & & \\
\hline $22, \mathrm{CC}$ & 95.02 & 1.95 & -0.31 & 2.07 & -0.55 & -2.22 & 1.50 & & \\
\hline $23, \mathrm{CC}$ & 96.35 & & & 1.95 & -0.13 & -2.65 & 1.47 & & \\
\hline $24, \mathrm{CC}$ & 101.55 & & & 1.89 & -0.13 & -2.14 & 1.52 & & \\
\hline $25, \mathrm{CC}$ & 106.54 & & & 2.07 & -0.57 & -2.16 & 1.15 & & \\
\hline $26, \mathrm{CC}$ & 110.98 & & & 1.89 & -0.51 & -1.80 & 1.21 & & \\
\hline \multicolumn{10}{|l|}{$503 \mathrm{~A}$} \\
\hline $27, \mathrm{CC}$ & 112.79 & & & 1.73 & -0.39 & -2.42 & 1.30 & & \\
\hline & & & & 1.94 & & -1.82 & & & \\
\hline 29 & 124. & & & 1.89 & -0.32 & -2.23 & 1.31 & & \\
\hline $31, \mathrm{CC}$ & 133.74 & & & 2.04 & -0.24 & -2.04 & 1.57 & & \\
\hline $32, \mathrm{CC}$ & 134.50 & & & 1.87 & -0.25 & -1.75 & 1.53 & 2.71 & -1.49 \\
\hline $33, \mathrm{CC}$ & 139.27 & & & $\begin{array}{l}1.65 \\
1.73\end{array}$ & $\begin{array}{l}0.72 \\
0.76\end{array}$ & -1.79 & 0.90 & & \\
\hline $34, \mathrm{CC}$ & 145.99 & & & 1.92 & -0.50 & -2.06 & 1.36 & 2.45 & -1.77 \\
\hline & 147.6 & & & 1.98 & & -2.35 & 1.90 & 2.46 & \\
\hline $36, \mathrm{CC}$ & 154.5 & & & 1.96 & -0.17 & -2.26 & 1.84 & 2.42 & -1.67 \\
\hline $37, \mathrm{CC}$ & 158.45 & & & 1.97 & -0.25 & -1.95 & 1.82 & $\begin{array}{l}2.45 \\
2.58\end{array}$ & $\begin{array}{l}-1.78 \\
-1.74\end{array}$ \\
\hline $38, \mathrm{CC}$ & 160.20 & & & 1.99 & -0.20 & -1.96 & 1.80 & 2.69 & -1.92 \\
\hline $39, \mathrm{CC}$ & 167.55 & & & 1.86 & -0.28 & -2.29 & 1.98 & 2.42 & -1 \\
\hline $40, \mathrm{CC}$ & 169.90 & & & 1.92 & -0.26 & -2.24 & 1.62 & $\begin{array}{l}2.54 \\
2.69\end{array}$ & $\begin{array}{l}-1.94 \\
-1.76\end{array}$ \\
\hline $41, \mathrm{C}$ & 177. & & & 2.00 & -0.30 & -2.49 & 1.38 & & \\
\hline \multirow[t]{2}{*}{$42, \mathrm{CC}$} & 181.64 & & & 1.96 & -0.66 & -2.17 & 0.98 & $\begin{array}{l}2.73 \\
2.85\end{array}$ & $\begin{array}{l}-1.86 \\
-1.49\end{array}$ \\
\hline & & & & & & & & $\begin{array}{l}2.89 \\
2.91\end{array}$ & $\begin{array}{l}-1.49 \\
-1.34\end{array}$ \\
\hline $43, \mathrm{C}$ & 185.59 & & & 1.85 & -0.50 & -1.88 & 1.24 & 2.20 & -1.92 \\
\hline & & & & & -0 & -2.21 & & 2.47 & -2.12 \\
\hline & & & & & -0 & -2 & & & -1 \\
\hline & 200.8 & & & 1.66 & -0.18 & -2.21 & 1.72 & 1.75 & -1.20 \\
\hline \multirow{2}{*}{$\begin{array}{l}47, \mathrm{CC} \\
48, \mathrm{C}\end{array}$} & 207.65 & & & 1.75 & 0.21 & -2.04 & 2.02 & 2.31 & -1.43 \\
\hline & & & & 1.8 & 0.1 & & & 2.65 & -1.15 \\
\hline 49 & 21 & & & 1.8 & -0 & -2.47 & 1.94 & & \\
\hline & & & & 1. & & -2.47 & & & -1.59 \\
\hline & 22 & & & 1. & 0. & -2.72 & 2.34 & 2.75 & -1.37 \\
\hline & & & & 1.92 & 0.24 & & & 2.81 & -0 \\
\hline $53, \mathrm{CC}$ & 229.90 & & & 1.82 & 0.66 & -2.20 & 2.45 & 2.35 & -0 \\
\hline \multirow[b]{2}{*}{$34, \mathrm{CC}$} & 234.62 & & & 1.79 & 0.49 & -2.22 & 1.95 & 2.46 & $\begin{array}{l}-0.70 \\
-1.13\end{array}$ \\
\hline & & & & & & -2.22 & & $\begin{array}{l}2.23 \\
2.06\end{array}$ & $\begin{array}{l}-1.13 \\
-0.62\end{array}$ \\
\hline
\end{tabular}

the initial growth of permanent Northern Hemisphere continental ice cover (3.2 Ma; Shackleton and Opdyke, 1977). Tables 5A and 5B present Atlantic-Pacific contrasts ( $\delta 502$ minus $\delta 503)$ and vertical contrasts ( $\delta$ benthic minus $\delta$ planktonic), respectively, calculated from the statistics in Table 4. Figures 3 and 4 are plots of Table 5 results. Results based on these time intervals are speculative, because: (1) neither site may be typical of its entire ocean basin, (2) there are sometimes few data points for intervals as long as $2 \mathrm{Ma}$, and (3) the observed contrasts are sometimes within the precision of analysis. Also, changes between the time intervals appear as "steps," when in fact they may be occurring gradually over millions of years.

\section{RESULTS AND DISCUSSION}

The late Miocene and Pliocene intervals up to about 3.2 $\mathrm{Ma}$ are marked by no net enrichments or depletions in ${ }^{18} \mathrm{O}$ and by less variability than in the late Pliocene (Table 4A). Similar trends have been previously noted by Shackleton and Kennett (1975), Shackleton and Opdyke (1977), Shackleton and Cita (1979), Keigwin (1979), Keigwin and Thunell (1979), and Keigwin et al. (1979). A general increase in the magnitude and variability in $\delta^{18} \mathrm{O}$ beginning at $3.2 \mathrm{Ma}$ reflects the growth of a permanent Northern Hemisphere continental ice cover and increased climatic variability. The overall variability in 


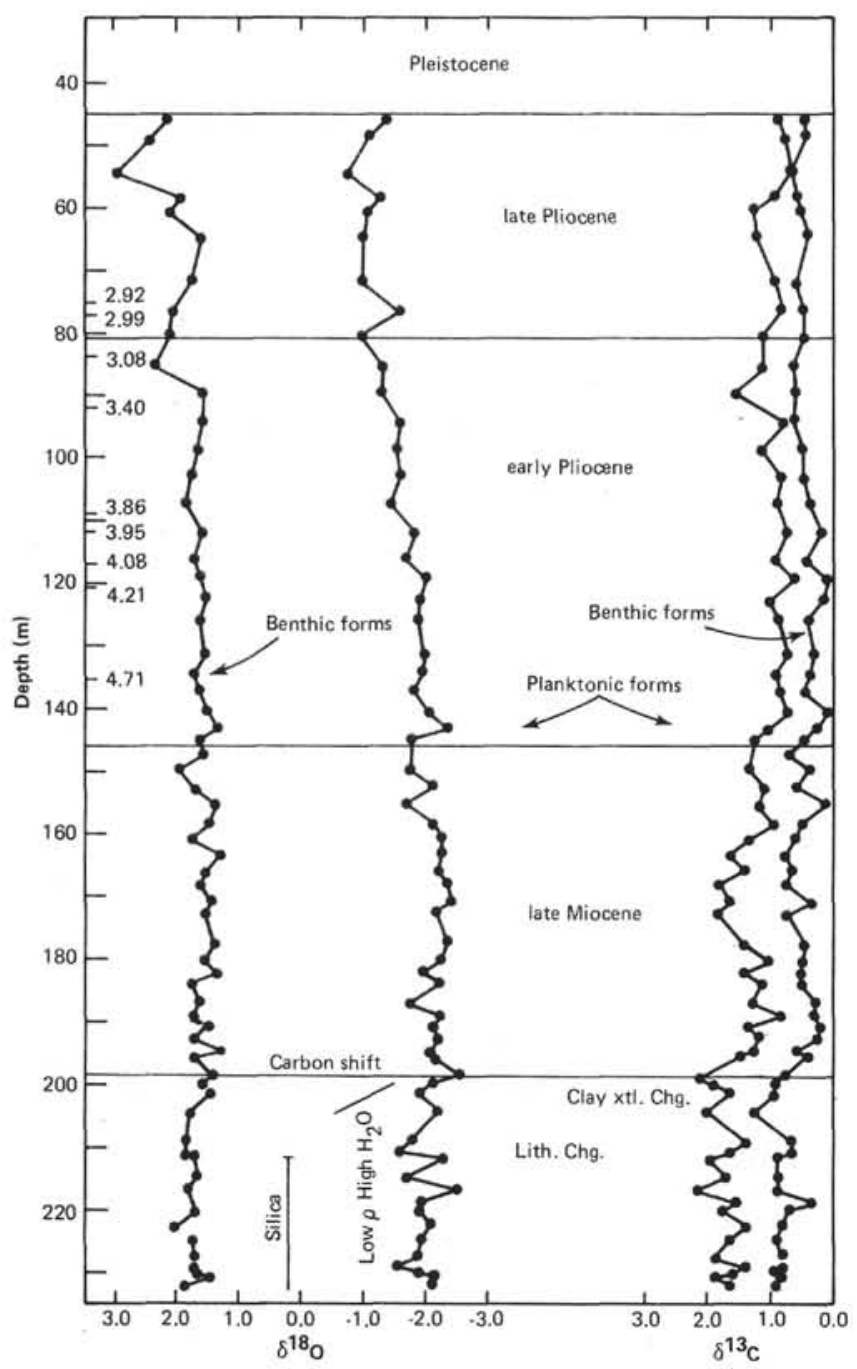

Figure 1. Stable isotope results (\%, PDB) at Colombian Basin DSDP Site 502. Absolute ages from magnetostratigraphy (Kent and Spariosu, this volume). Benthic oxygen isotopes reveal little change before the inferred growth of permanent Northern Hemisphere continental ice cover at about $3.2 \mathrm{Ma}$ at this site as well as Site 503 (Fig. 2). Planktonic foraminifers become gradually enriched in 180 beginning about $4.2 \mathrm{Ma}$, which may reflect an increase in North Atlantic surface-water salinity. Carbon isotopes record the late Miocene $\delta^{13} \mathrm{C}$ shift at about 200 meters $(6 \mathrm{Ma})$, closely associated with other physical and sedimentological changes at the site.

benthic foraminiferal $\delta^{18} \mathrm{O}$ is slightly greater in the Atlantic site than the Pacific in the late Miocene and late Pliocene (Table 4A). Shackleton and Cita (1979) noted greater Atlantic $\delta^{18} \mathrm{O}$ variability, when comparing Atlantic Ocean DSDP Site 397 results to unpublished results from equatorial Pacific piston core RC12-66. Moore et al. (in prep.) have synthesized Cenozoic isotope data, and these workers also find greater $\delta^{18} \mathrm{O}$ variability in Atlantic benthic foraminifers since the late Miocene. Shackleton and Cita (1979) attribute this greater variability to greater changes in Atlantic deep water temperatures relative to those of the Pacific, whereas Moore et al. (in preparation) consider the relative importance in the Atlantic of water masses having differing isotopic composition.

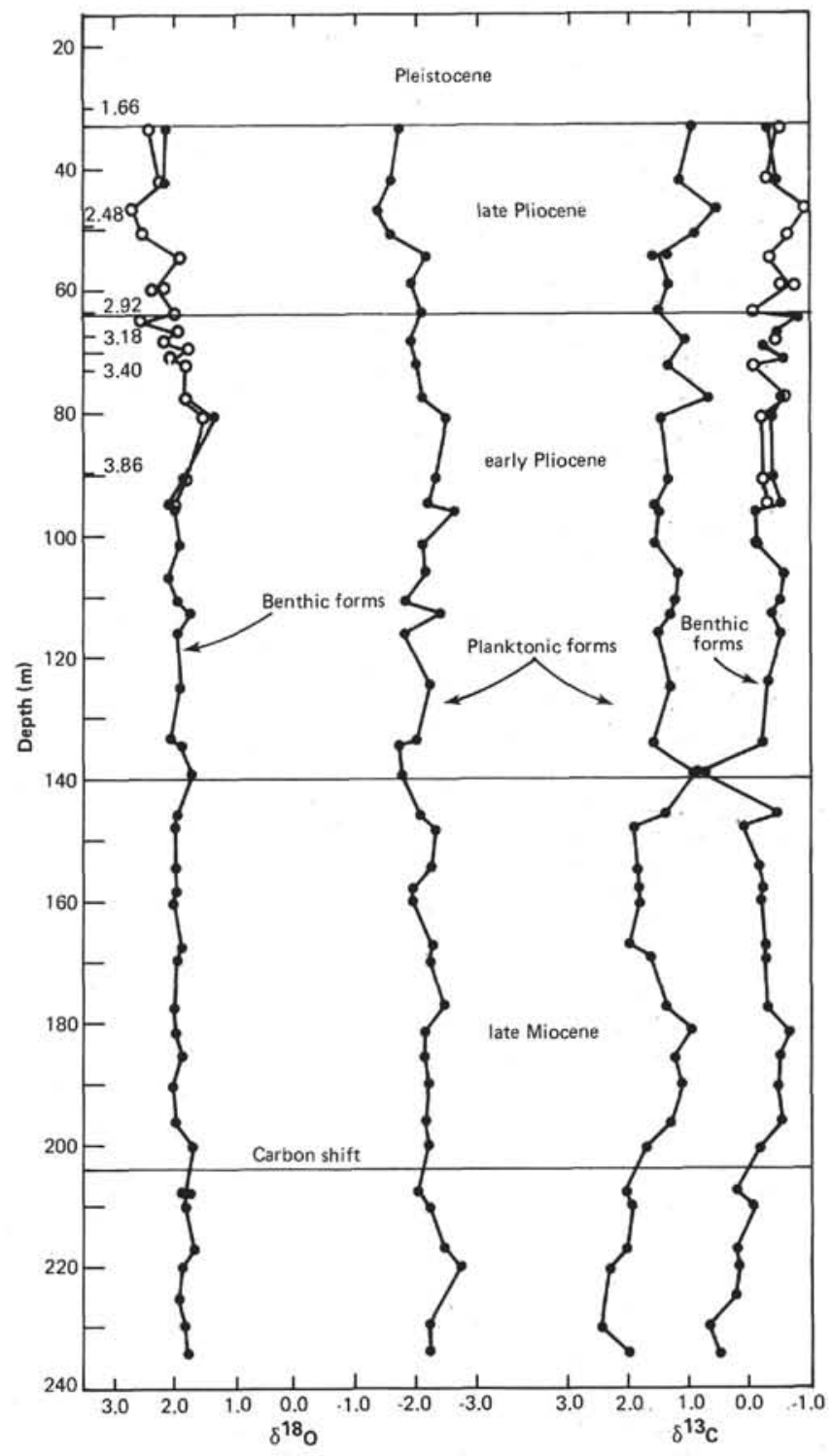

Figure 2. Stable isotope results (\%, PDB) at eastern equatorial Pacific DSDP Site 503. Absolute ages from magnetostratigraphy (Kent and Spariosu, this volume). Oxygen isotopes reveal the inferred growth of permanent Northern Hemisphere continental ice cover at about 3.2 Ma. Carbon isotopes record the late Miocene $\delta^{13} \mathrm{C}$ shift at about 205 meters (6 Ma).

Several interesting changes occur in the Site 502 sedimentary column below about 220 meters (Fig. 1). These include: the last occurrence of rare siliceous microfossils at 211 meters (site chapter 502; Riedel and Westberg, this volume); a change in the sediment's physical properties from low bulk density and high water content below 203 meters to high bulk density and low water content above (Mayer, this volume); a lithological change from ashbearing clays to foraminifer-bearing nannofossil marls at 211 meters (site chapter 502); and a change from low to high clay mineral crystallinity at 203 meters (Zimmerman, this volume). Low bulk density, high water content, low clay mineral crystallinity, and the presence of siliceous microfossils are characteristic of Pacific Ocean sediments today. There also appears to be a change in 
Table 3. Paired analyses of C. wuellerstorfi and C. kullenbergi compiled from Table 2 .

\begin{tabular}{|c|c|c|c|c|c|c|c|}
\hline \multirow[b]{2}{*}{ Sample } & \multicolumn{2}{|c|}{$\begin{array}{c}C . \\
\text { wuellerstorfi }\end{array}$} & \multicolumn{2}{|c|}{$\begin{array}{c}\text { C. } \\
\text { kullenbergi }\end{array}$} & \multicolumn{2}{|c|}{ C. $w .-C . k$. } & \multirow[b]{2}{*}{$\bar{x}$ and $o$ of $C . w .-C . k$} \\
\hline & ${ }_{\delta^{18} \mathrm{O}}$ & ${ }_{8^{13}} \mathrm{C}$ & ${ }^{18} \mathrm{O}$ & ${ }_{\delta^{13}} \mathrm{C}$ & ${ }_{\delta^{18}}^{18} \mathrm{O}$ & ${ }^{13} \mathrm{C}$ & \\
\hline \multicolumn{8}{|l|}{ 502A } \\
\hline $67, \mathrm{CC}$ & 1.72 & 0.90 & 1.60 & 0.75 & +0.12 & +0.15 & \\
\hline \multicolumn{8}{|l|}{$502 \mathrm{C}$} \\
\hline $29, \mathrm{CC}$ & $1.74^{\circ}$ & $0.41^{*}$ & 1.65 & 0.45 & +0.09 & -0.04 & \\
\hline $30, \mathrm{CC}$ & 1.74 & 0.73 & 1.79 & 0.75 & -0.05 & -0.02 & \\
\hline $31, \mathrm{CC}$ & 2.04 & 0.82 & 1.75 & 0.56 & +0.29 & +0.26 & \\
\hline $32, \mathrm{CC}$ & 1.75 & 0.92 & 1.81 & 0.74 & -0.06 & +0.18 & $\Delta \delta^{18} \mathrm{O}=+0.03 \pm 0.14$ \\
\hline $34, \mathrm{CC}$ & 1.74 & 0.84 & 1.73 & 0.81 & +0.01 & +0.03 & $\Delta \delta^{13} \mathrm{C}=+0.10 \pm 0.12$ \\
\hline $35, \mathrm{CC}$ & 1.68 & 0.91 & 1.57 & 0.95 & +0.11 & -0.04 & \\
\hline $36, \mathrm{CC}$ & 1.53 & 0.88 & 1.76 & 0.73 & -0.23 & +0.15 & \\
\hline $37, \mathrm{CC}$ & 1.86 & 0.92 & 1.83 & 0.67 & +0.03 & +0.25 & \\
\hline \multicolumn{8}{|l|}{ 503B } \\
\hline $8, \mathrm{CC}$ & 2.39 & -0.48 & 2.17 & -0.31 & +0.22 & -0.17 & \\
\hline $10, \mathrm{CC}$ & $2.20^{\circ}$ & $-0.35^{*}$ & 2.23 & -0.42 & -0.03 & +0.07 & \\
\hline $18, \mathrm{CC}$ & 1.77 & -0.58 & 1.76 & -0.56 & +0.01 & -0.02 & $\Delta \delta^{18} \mathrm{O}=+0.04 \pm 0.13$ \\
\hline $19, \mathrm{CC}$ & 1.98 & -0.20 & 1.80 & -0.37 & +0.18 & +0.17 & $\Delta \delta^{13} \mathrm{C}=+0.07 \pm 0.15$ \\
\hline $21, \mathrm{CC}$ & 1.80 & -0.24 & 1.84 & -0.38 & -0.04 & +0.14 & \\
\hline $22, \mathrm{CC}$ & 1.95 & -0.31 & 2.07 & -0.55 & -0.12 & +0.24 & \\
\hline
\end{tabular}

Note: ${ }^{\bullet}=$ Average of two analyses.

Table 4A. Simple statistics of oxygen isotope data from Table 2 for four Neogene time intervals.

\begin{tabular}{|c|c|c|c|c|}
\hline Time Interval & G. quadrilobatus & C. wuellerstorfi & C. kullenbergi & O. tener \\
\hline \multicolumn{5}{|l|}{ Site 502} \\
\hline $\begin{array}{l}\text { Post-ice growth } \\
\text { (less than } \sim 3 \mathrm{Ma}) \\
(45.69-85.46 \mathrm{~m}) \\
\text { Pre-ice growth } \\
(\sim 3-5 \mathrm{Ma}) \\
(89.56-145.14 \mathrm{~m}) \\
\text { Post-carbon shift } \\
(\sim 5-6 \mathrm{Ma}) \\
(147.49-195.64 \mathrm{~m}) \\
\text { Pre-carbon shift } \\
(\sim 6 \text { to } 8 \mathrm{Ma}) \\
(198.97-227.81 \mathrm{~m})\end{array}$ & $\begin{aligned} n & =10 \\
\bar{x} & =-1.13 \\
\sigma & =0.24 \\
n & =16 \\
\bar{x} & =-1.77 \\
\sigma & =0.26 \\
n & =20 \\
\bar{x} & =-2.11 \\
\sigma & =0.20 \\
n & =18 \\
\bar{x} & =-1.96 \\
\sigma & =0.27\end{aligned}$ & $\begin{array}{l}10 \\
2.14 \\
0.37 \\
16 \\
1.60 \\
0.11 \\
21 \\
1.57 \\
0.18 \\
18 \\
1.72 \\
0.15\end{array}$ & $\begin{array}{l}- \\
\overline{-} \\
\overline{-} \\
\overline{-} \\
\overline{-} \\
\overline{9} \\
1.72 \\
0.09\end{array}$ & $\begin{array}{l}- \\
z \\
- \\
- \\
\overline{7} \\
2.24 \\
0.13 \\
6 \\
2.21 \\
0.11\end{array}$ \\
\hline \multicolumn{5}{|l|}{ Site 503} \\
\hline $\begin{array}{l}\text { Post-ice growth } \\
\text { (less than } \sim 3 \mathrm{Ma}) \\
(33.52-64.90 \mathrm{~m}) \\
\text { Pre-ice growth } \\
(\sim 3-5 \mathrm{Ma}) \\
(66.80-134.50 \mathrm{~m}) \\
\text { Post-carbon shift } \\
(\sim 5-6 \mathrm{Ma}) \\
(139.27-200.81 \mathrm{~m}) \\
\text { Pre-carbon shift } \\
(\sim 6-8 \mathrm{Ma}) \\
(207.65-234.62 \mathrm{~m})\end{array}$ & $\begin{aligned} \frac{n}{\bar{x}} & =7 \\
\sigma & =0.30 \\
n & =15 \\
\bar{x} & =-2.14 \\
\sigma & =0.26 \\
n & =14 \\
\bar{x} & =-2.14 \\
\sigma & =0.19 \\
n & =6 \\
\bar{x} & =-2.35 \\
\sigma & =0.25\end{aligned}$ & $\begin{array}{l}7 \\
2.28 \\
0.28 \\
6 \\
1.90 \\
0.15 \\
- \\
- \\
- \\
- \\
- \\
-\end{array}$ & $\begin{array}{l}3 \\
2.30 \\
0.18 \\
16 \\
1.91 \\
0.10 \\
14 \\
1.91 \\
0.11 \\
7 \\
1.81 \\
0.07\end{array}$ & $\begin{array}{l}- \\
\overline{-} \\
- \\
- \\
\overline{-} \\
13 \\
2.49 \\
0.28 \\
7 \\
2.43 \\
0.27\end{array}$ \\
\hline
\end{tabular}

the Site 502 benthic isotope data close to these other changes (around $205 \mathrm{~m}$ ). The lowermost 15 benthicforaminiferal oxygen isotope values from Site 502 are lighter than the overlying late Miocene and early Pliocene values by a statistically significant $0.20 \%\left(T_{53}=\right.$ $\left.4.65 ; t_{53} ; 1-\alpha=1.68\right)$. In the lower 30 to 40 meters of each section (approximately the "pre-carbon shift" time interval, Tables $4 \mathrm{~A}$ and $5 \mathrm{~A}$ ), the $\delta^{18} \mathrm{O}$ of benthic forms from each site is nearly identical, whereas in more recent intervals the Atlantic site is always isotopically lighter by a few tenths $\%$. The similarities in benthic $\delta^{18} \mathrm{O}$, physical properties, and sedimentological characteristics below about 200 meters at each site suggest that prior to about $6 \mathrm{Ma}$ the Colombian Basin had some $\mathrm{Pa}$ cific Ocean affinity, although carbon isotope evidence (see following discussion) indicates that deep waters in each basin were largely segregated by this time.
Table 4B. Simple statistics of carbon isotope data from Table 2 for four Neogene time intervals.

\begin{tabular}{|c|c|c|c|c|}
\hline Time Interval & G. quadrilobatus & C. wuellerstorfi & C. kullenbergi & O. tener \\
\hline \multicolumn{5}{|l|}{ Site 502} \\
\hline \multirow[t]{2}{*}{ Post-ice growth } & $n=10$ & 10 & - & - \\
\hline & $\begin{array}{l}\bar{x}=0.98 \\
\sigma=0.21\end{array}$ & 0.53 & $\bar{z}$ & $\bar{z}$ \\
\hline \multirow[t]{3}{*}{ Pre-ice growth } & $n=16$ & 16 & - & - \\
\hline & $\bar{x}=0.93$ & 0.37 & - & - \\
\hline & $\sigma=0.25$ & 0.17 & - & \\
\hline \multirow[t]{3}{*}{ Post-carbon shift } & $n=20$ & 21 & - & 7 \\
\hline & $\bar{x}=1.36$ & 0.50 & - & -1.43 \\
\hline & $\sigma=0.26$ & 0.19 & - & 0.09 \\
\hline \multirow[t]{3}{*}{ Pre-carbon shift } & $n=18$ & 18 & 9 & 6 \\
\hline & $\bar{x}=1.77$ & 0.85 & 0.71 & -0.43 \\
\hline & $\sigma=0.25$ & 0.17 & 0.14 & 0.21 \\
\hline \multicolumn{5}{|l|}{ Site 503} \\
\hline \multirow[t]{2}{*}{ Post-ice growth } & $n=?$ & 7 & 3 & - \\
\hline & $\begin{aligned} \bar{x} & =1.12 \\
g & =0.34\end{aligned}$ & -0.48 & $\begin{array}{r}-0.50 \\
0.23\end{array}$ & $\bar{z}$ \\
\hline \multirow[t]{3}{*}{ Pre-ice growth } & $\begin{array}{l}\sigma=0.34 \\
n=15\end{array}$ & 6.29 & 16 & - \\
\hline & $\bar{x}=1.33$ & -0.31 & -0.39 & - \\
\hline & $\sigma=0.23$ & 0.17 & 0.15 & - \\
\hline \multirow[t]{3}{*}{ Post-carbon shift } & $n=14$ & - & 14 & 13 \\
\hline & $\bar{x}=1.50$ & - & -0.25 & -1.70 \\
\hline & $\sigma=0.36$ & - & 0.34 & 0.23 \\
\hline \multirow[t]{3}{*}{ Pre-carbon shift } & $n=6$ & - & 7 & 7 \\
\hline & $\bar{x}=2.12$ & - & 0.27 & -1.15 \\
\hline & $\sigma=0.22$ & - & 0.24 & 0.30 \\
\hline
\end{tabular}

Table 5A. Summary of geographic contrasts in isotopes ( $\delta 502$ minus 8503 ) from data in Tables 2 and 4.

\begin{tabular}{lcccc}
\hline Time Interval & $\begin{array}{c}\text { Benthic } \\
\delta^{13} \mathrm{C}\end{array}$ & $\begin{array}{c}\text { Benthic } \\
\delta^{18} \mathrm{O}\end{array}$ & $\begin{array}{c}\text { Planktonic } \\
\delta^{13} \mathrm{C}\end{array}$ & $\begin{array}{c}\text { Planktonic } \\
\delta^{18} \mathrm{O}\end{array}$ \\
\hline Core top & $0.98 \%$ & $-0.57 \%$ & - & - \\
Post-ice growth & $1.01 \%$ & $-0.14 \%$ & $-0.14 \%$ & $0.66 \%$ \\
Pre-ice growth & $0.76 \%$ & $-0.30 \%$ & $-0.40 \%$ & $0.37 \%$ \\
Post- $\delta^{13} \mathrm{C}$ shift & $0.75 \%$ & $-0.34 \%$ & $-0.14 \%$ & $0.03 \%$ \\
Pre- $\delta^{13} \mathrm{C}$ shift & $0.58 \%$ & $-0.09 \%$ & $-0.35 \%$ & $0.39 \%$ \\
\hline
\end{tabular}

Table 5B. Summary of vertical isotopic contrasts ( $\delta$ planktonic - $\delta$ benthic) from data in Table 4.

\begin{tabular}{lcccc}
\hline & \multicolumn{2}{c}{ Site 502} & \multicolumn{2}{c}{ Site 503} \\
\multicolumn{1}{c}{ Time Slab } & $\delta^{18} \mathrm{O}$ & $\delta^{15 \mathrm{C}}$ & $\delta^{18} \mathrm{O}$ & $\delta^{13} \mathrm{C}$ \\
\hline Post-ice growth & -3.27 & +0.45 & -4.07 & +1.60 \\
Pre-ice growth & -3.37 & +0.56 & -4.05 & +1.72 \\
Post- $\delta^{13} \mathrm{C}$ shift & -3.68 & +0.86 & -4.05 & +1.75 \\
Pre- $\delta^{13} \mathrm{C}$ shift & -3.68 & +0.92 & -4.16 & +1.85 \\
\hline
\end{tabular}

The most striking carbon isotope result of each time series is the late Miocene carbon isotope shift in benthic and planktonic foraminifers (Figs. 1 and 2). This negative change in $\delta^{13} \mathrm{C}$ has been found to be a useful stratigraphic marker at many locations throughout the IndoPacific (Keigwin, 1978, 1979; Loutit and Kennett, 1979; Bender and Keigwin, 1979; Vincent et al., 1980; Haq et al., 1980; Keigwin and Shackleton, 1980). It has been paleomagnetically dated in the upper reversed interval of paleomagnetic Chron 6 (Loutit and Kennett, 1979; Keigwin and Shackleton, 1980), which gives the age of 6 $\mathrm{Ma}$ (Kent and Spariosu, this volume). The carbon shift is closely associated in time with the first occurrence of the nannofossil Amaurolithus primus (Haq et al., 

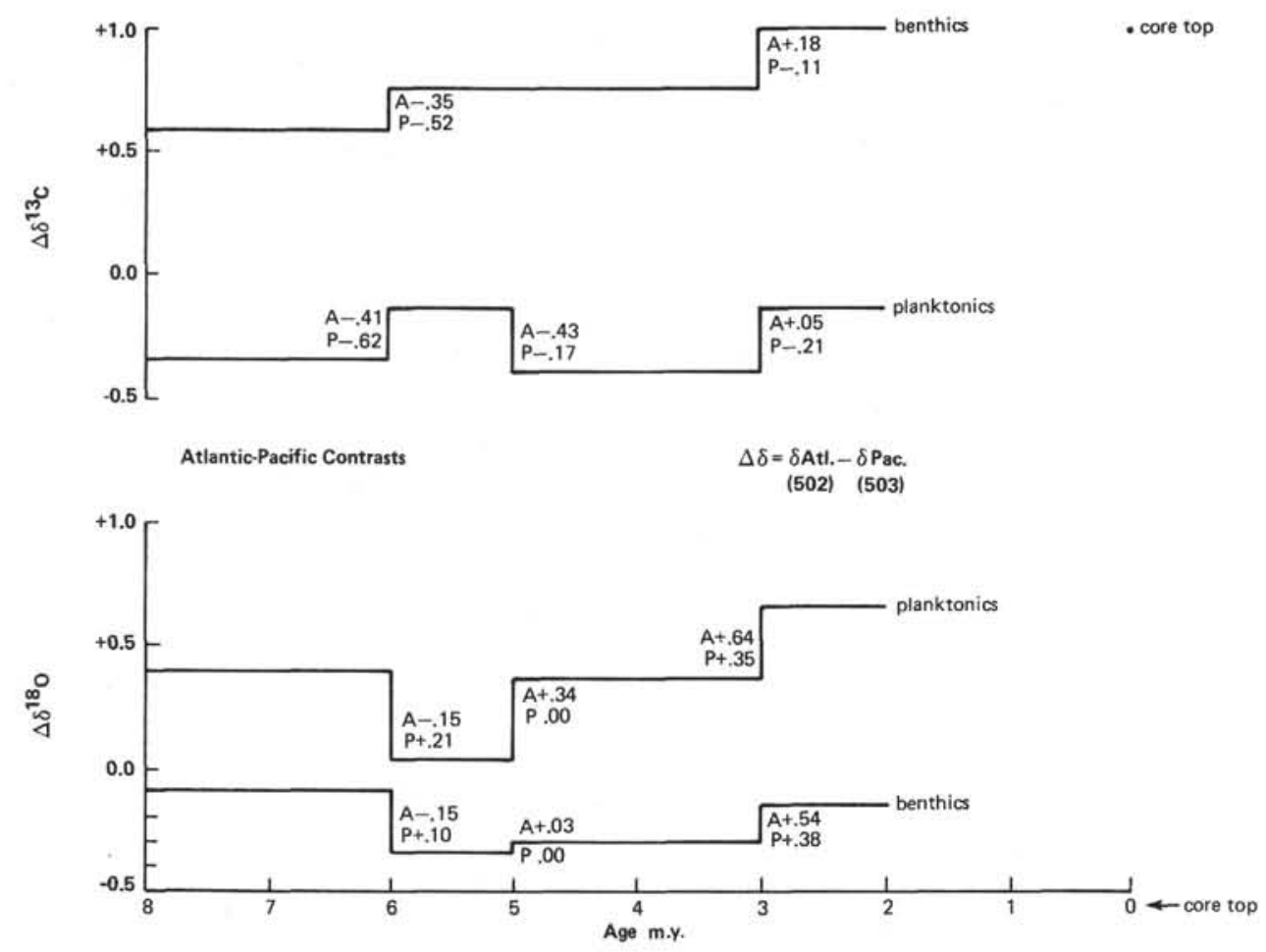

Figure 3. The history of geographic isotope contrasts $(\delta 502$ minus $\delta 503)$ calculated for four stratigraphic intervals from data in Tables 2 and 4 . Numbers labeled $A$ and $P$ show the magnitude of Atlantic and Pacific isotope change going forward in time between each interval. Benthic $\Delta \delta^{13} \mathrm{C}$ shows the stepwise growth to modern values of the Atlantic-Pacific deep water $\delta^{13} \mathrm{C}$ contrast, which is maintained by the net flow of deep waters from the North Atlantic to the North Pacific. The interesting planktonic $\Delta \delta^{13} \mathrm{C}$ pattern is unexplained. The history of the contrast in Atlantic-Pacific planktonic $\delta^{18} \mathrm{O}$ is dominated by large Pliocene increases of Atlantic planktonic foraminiferal $\delta^{18} \mathrm{O}$, which may reflect increasing North Atlantic surface-water salinity.

1980). This datum is found in Sections 502A-59, CC and $503-48, \mathrm{CC}$, so this change in Leg 68 sites probably occurred $6 \mathrm{Ma}$.

The cause of the carbon shift is still unclear. Bender and Keigwin (1979) suggested changing circulation and upwelling patterns as the cause, possibly including an increase in basin-basin fractionation of nutrients (Berger, 1970) by reduction of deep-water communication between the Atlantic and Pacific at the present location of the Isthmus of Panama. In addition, Vincent et al., (1980) proposed the change of foraminiferal "vital effects,' a late Miocene sea-level fall, and climatic change as contributing factors. Although the $\delta^{13} \mathrm{C}$ shift was associated with a late Miocene regression and the "Messinian event" (Keigwin, 1978), it could not have been a simple result of Mediterranean dessication because the Messinian evaporites are depleted in ${ }^{13} \mathrm{C}$ (Lloyd and Hsü, 1973). Likewise the $\delta^{13} \mathrm{C}$ of foraminiferal calcite is not a simple function of sea level but can be related to this because it correlates inversely with $\delta^{18} \mathrm{O}$ (especially for benthic foraminifers) in the Quaternary (Broecker, in press) and in the pre-Quaternary (Loutit, in prep.).

Results of carbon isotope analysis suggest that lowlatitude deep waters of the Atlantic and Pacific were significantly isolated by 6 to $8 \mathrm{Ma}$ and that the extent of this segregation has increased since then. In the modern ocean the net flow of deep waters from the North Atlan- tic to the North Pacific and the subsequent increase in organic-matter oxidation and $\mathrm{CaCO}_{3}$ dissolution (basin-basin fractionation, Berger, 1970) results in a $1.2 \%$ depletion in ${ }^{13} \mathrm{C}$ of deep Pacific waters relative to deep Atlantic waters (Kroopnick et al., 1972). Comparing North Atlantic hydrocasts (Kroopnick et al., 1972; Kroopnick, 1980) to east equatorial Pacific hydrocasts (Kroopnick, 1974) shows the basin-basin contrast to be closer to $1 \%$ at the locations of Sites 502 and 503. The history of this $\delta^{13} \mathrm{C}$ contrast, based on data from Sites 502 and 503, is summarized in Table 5A and Figure 3. The present-day benthic $\delta^{13} \mathrm{C}$ contrast at these two locations (based on core tops, Table 2) is $0.98 \%$ (in excellent agreement with Kroopnick's data), whereas in the precarbon-shift interval it was $0.60 \%$ (Table 5A, Fig. 3).

That the pre-carbon-shift $\Delta \delta^{13} \mathrm{C}$ was as large as $0.60 \%$ suggests that even though the $\Delta \delta^{18} \mathrm{O}$ was small $(0.1 \%)$, the deep tropical Atlantic and Pacific oceans were significantly isolated by $6 \mathrm{Ma}$. Figure 3 shows that the geographic contrast in benthic $\delta^{13} \mathrm{C}$ has increased in two steps since about $8 \mathrm{Ma}$. The first step occurred at the carbon shift $(6 \mathrm{Ma})$ and results from a greater negative change in Pacific deep water than Atlantic. This was noted at other locations and cited as possible evidence for an increase in basin-basin fractionation as a result of uplift of a sill at Panama above some unspecified critical depth (Bender and Keigwin, 1979). Other inde- 


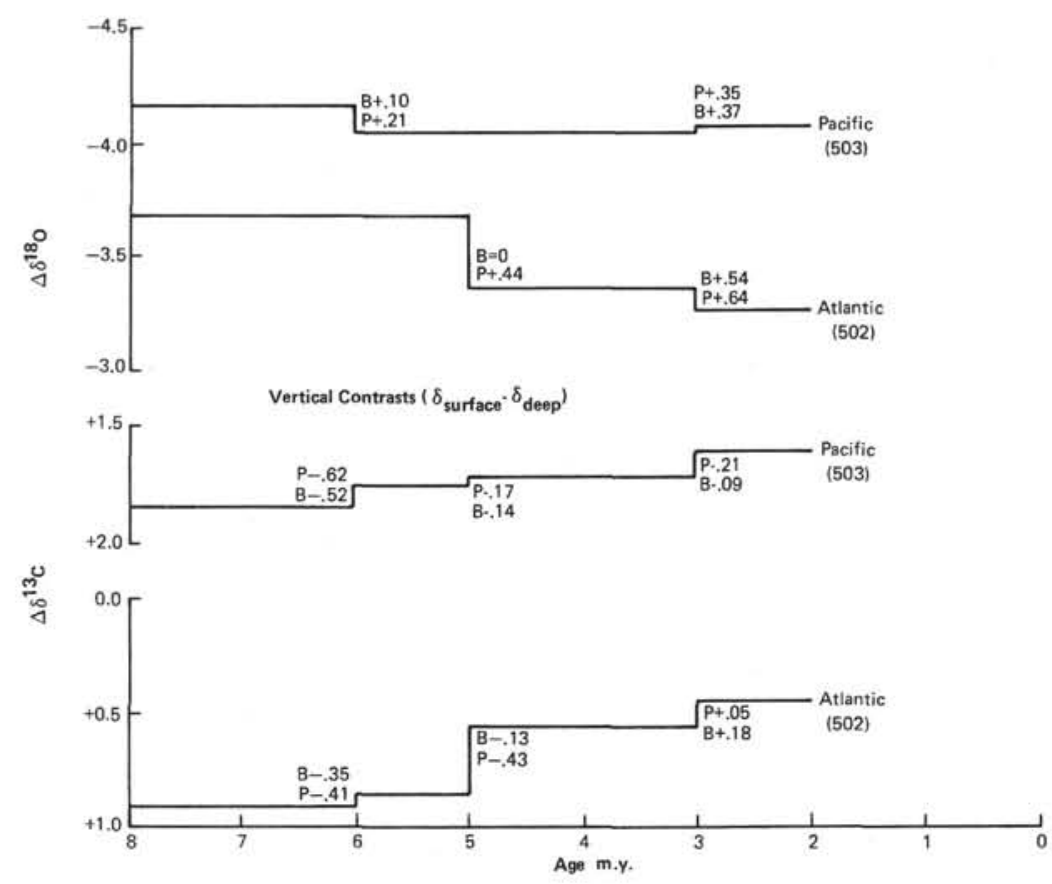

Figure 4. The history of vertical isotope contrasts ( $\delta$ planktonic minus $\delta$ benthic) calculated for four stratigraphic intervals from data in Table 4. Numbers labeled $P$ and B show the magnitude of the change in isotopes in planktonic and benthic forms going forward in time between each time interval. The Pliocene vertical oxygen isotope contrast decreases markedly in the Atlantic $\left(\Delta \delta^{18} \mathrm{O}\right.$ increases) but not in the Pacific as a result of enrichment in ${ }^{18} \mathrm{O}$ of Site 502 planktonic foraminifers. This may reflect increasing North Atlantic surface-water salinity. At both locations the vertical carbon contrast decreases, which may indicate a change in chemistry of the oceans.

pendent evidence for increased basin-basin fractionation is the crossover of Atlantic and Pacific CCDs in the late Miocene (van Andel, 1975) which heralds the origins of modern abyssal circulation. The $3 \mathrm{Ma}$ step in the geographic $\delta^{13} \mathrm{C}$ contrast brought the benthic $\Delta \delta^{13} \mathrm{C}$ to modern values. At this time benthic $\delta^{13} \mathrm{C}$ increased in the Atlantic and decreased in the Pacific. Biogeographic evidence suggests that by $3 \mathrm{Ma}$ there was only the shallowest marine connection between the Atlantic and the Pacific (Keigwin, this volume), so basin-basin fractionation of deep waters by the emergent isthmus was probably already at its maximum. I speculate that the second $\Delta \delta^{13} \mathrm{C}$ increase may have resulted from increased production of North Atlantic Deep Water (NADW), which is marked by high dissolved oxygen and total $\mathrm{CO}_{2}$ enriched in ${ }^{13} \mathrm{C}$. Blanc et al. (1980) have examined the history of NADW production by comparing carbon isotopes in Neogene benthic foraminifers between the North Atlantic and South Pacific. They conclude that NADW formation began late in the middle Miocene because at that time $\delta^{13} \mathrm{C}$ of North Atlantic benthic foraminifers increased to Holocene values, but no such increase was seen in the South Pacific. Unfortunately there is too little detail in the Blanc et al. (1980) study to define middle Pliocene interocean changes in $\delta^{13} \mathrm{C}$. Regardless of the exact cause of the two $\Delta \delta^{13} \mathrm{C}$ steps in the present study, the benthic foraminiferal $\delta^{13} \mathrm{C}$ at Sites 502 and 503 indicates that deep-sea circulation achieved essentially the modern pattern by about $3 \mathrm{Ma}$.
Planktonic foraminiferal carbon generally follows the trends in benthic foraminifers (Figs. 1 and 2); thus, these records probably reflect variability in global phenomena such as climate, sea level, circulation, and upwelling. The close correspondence between benthic and planktonic records is especially apparent at the late Miocene carbon shift; the change in planktonic forms is -0.41 and $-0.62 \%$ at Sites 502 and 503 , respectively, and benthic forms change by -0.35 and $-0.52 \%$ (Table 4B). Examination of the vertical isotope contrasts ( $\delta$ planktonic minus $\delta$ benthic) at each location reveals a decrease in $\Delta \delta^{13} \mathrm{C}$ between about 8 and $3 \mathrm{Ma}$ (Fig. 4). At the Atlantic site the total change during this time interval is $-0.47 \%$, nearly twice that in the Pacific $(-0.25 \%)$. Broecker (1974; Broecker, in press) suggests that changes in $\Delta \delta^{13} \mathrm{C}$ may reflect changes in ocean chemistry, because the isotopic separation of benthic and planktonic carbon is related to the carbon-to-phosphorus ratio in the ocean. Since the total change differs by a factor of two between Sites 502 and 503 there must be local or basinwide effects in the Atlantic as well as potential oceanwide chemistry changes. More recently, Broecker (in press) suggests that benthic foraminiferal $\delta^{13} \mathrm{C}$ may decrease during glacial stages (at least for the late Quaternary) due to sea-level lowering and increased phosphate in the ocean. Planktonic foraminiferal $\delta^{13} \mathrm{C}$ generally shows little change in the Quaternary, so the $\Delta \delta^{13} \mathrm{C}$ record should increase during glacial times. Although the late Neogene is marked by increasing glacia- 
tion and falling sea level, the vertical $\delta^{13} \mathrm{C}$ contrast clearly decreases because between each stratigraphy interval (going forward in time) the planktonic change in $\delta^{13} \mathrm{C}$ is relatively more negative than the benthic change (Fig. 4). More data from other ocean basins are necessary to interpret $\Delta \delta^{13} \mathrm{C}$ records with confidence.

Figure 5 presents planktonic records from Sites 502 and 503 as a function of age from the late Miocene carbon shift, which I consider to be the oldest well-dated horizon in these cores (6 Ma). Ages are interpolated between the Miocene/Pliocene boundary $(5.26 \mathrm{Ma})$, the carbon shift, and paleomagnetic reversals (Kent and Spariosu, this volume). The planktonic foraminifers at both sites show evidence of several hundred thousand years' cyclicity in the latest Miocene. This occurs where samples are most closely spaced in time and results nearly overlap, although at other times plankton results clearly differ (Fig. 5). This is also shown in Figure 3, which illustrates that the geographic contrast in planktonic carbon curiously decreases following each of the two step-

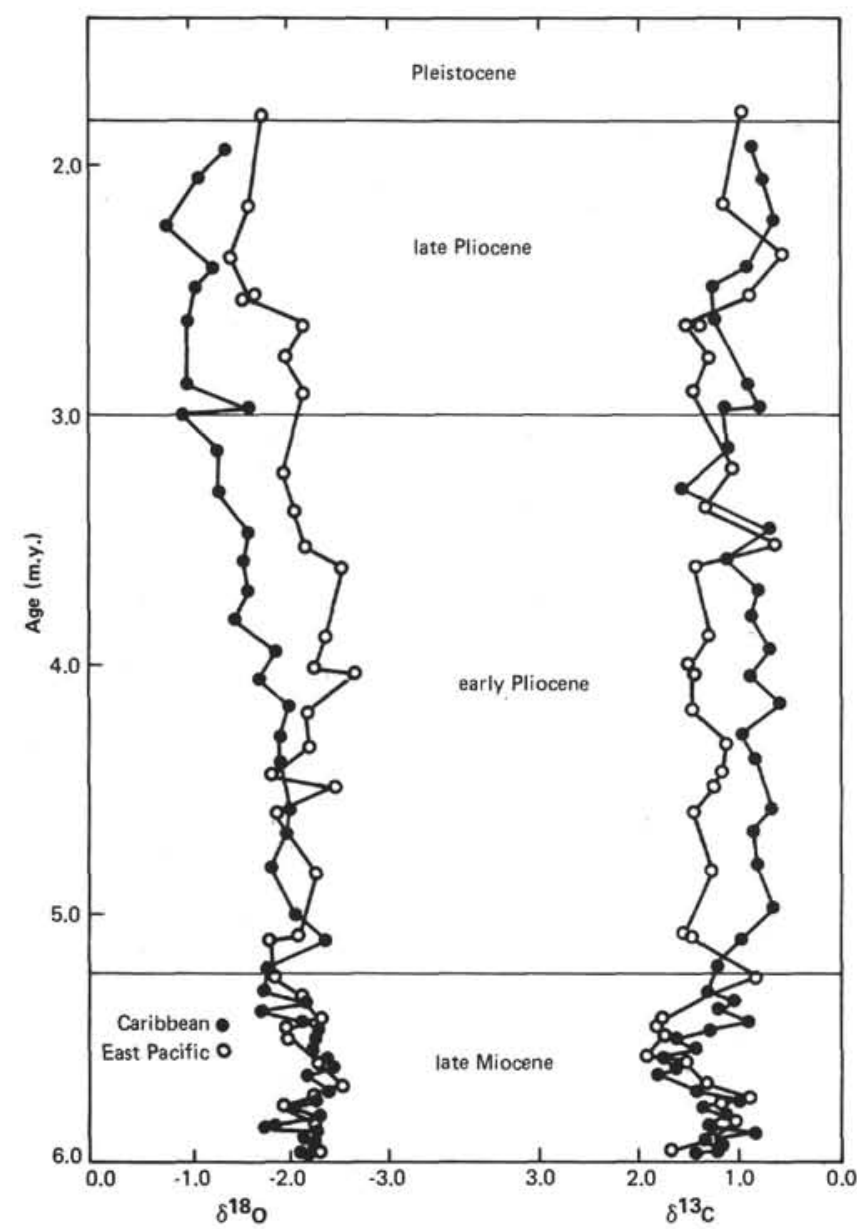

Figure 5. Post-carbon shift results of planktonic foraminifers from Sites $\mathbf{5 0 2}$ and 503 versus absolute age. Ages calculated from magnetostratigraphy (Kent and Spariosu, this volume), assuming the Miocene/Pliocene boundary to be $5.26 \mathrm{Ma}$ and the $\delta^{13} \mathrm{C}$ shift to be $6 \mathrm{Ma}$. The divergence of $\delta^{18} \mathrm{O}$ values beginning at $4.2 \mathrm{Ma}$ may reflect increasing North Atlantic surface-water salinity. This may have resulted from restricted surface-water communication between the Atlantic and Pacific, stemming from the emergent Panama Isthmus. wise increases in the benthic geographic contrast. It would be interesting to see if this pattern continues into the Quaternary. The details in planktonic-carbon isotope records are still poorly understood because of effects such as local surface water productivity, upwelling (Berger et al., 1978a; Prell and Curry, 1981), depth habitat, and vital effects (Williams et al., 1977; Berger et al., 1978b), in addition to the previously cited regional and global effects.

Analysis of oxygen isotopes from planktonic foraminifers, like carbon, isotopes, shows trends similar to those of benthic foraminifers. This is most obvious in the late Pliocene, where there is an oceanwide enrichment in ${ }^{18} \mathrm{O}$ resulting from growth of permanent Northern Hemisphere continental ice beginning at $3.2 \mathrm{Ma}$ (Figs. 1 and 2; Shackleton and Opdyke, 1977). In this respect, data from Site 503 are typical of records from the Neogene Pacific and the Quaternary worldwide; although in this study point-for-point correlations are complicated by sampling intervals as large as $100,000 \mathrm{y}$. The oxygen isotope record of planktonic foraminifers at Site $\mathbf{5 0 2}$ is the first late Neogene record from the Atlantic Ocean. A dramatic change begins in the early Pliocene, when average $\delta^{18} \mathrm{O}$ increases by 0.5 to $1.0 \%$ (Fig. 1). This is a time when benthic foraminifers show no net change, so an effect stemming from ice volume can be discounted. The history of geographic and vertical isotope contrasts also illustrate this change. The vertical oxygen isotope contrast at Site $\mathbf{5 0 3}$ has remained essentially unchanged for $8 \mathrm{~m}$.y., whereas it has decreased (contrast decreased, $\Delta \delta^{18} \mathrm{O}$ increased) at Site 502 due to the planktonic $\delta^{18} \mathrm{O}$ increase (Fig. 4). The geographic contrast in surface water $\delta^{18} \mathrm{O}$ decreased across the carbon shift and then increased up to the late Pliocene (Fig. 3). The two increases in $\Delta \delta^{18} \mathrm{O}$ that occurred after $6 \mathrm{Ma}$ result from a relative enrichment in ${ }^{18} \mathrm{O}$ of Site 502 planktonic foraminifers over those from Site 503. The plot of individual data points versus age reveals that most of the increase at Site 502 occurred in the middle Pliocene, beginning about $4.2 \mathrm{Ma}$ (Fig. 5). This is the time when the provinciality of tropical Atlantic and $\mathrm{Pa}$ cific planktonic foraminifers began (Keigwin, this volume). Since there is no faunal evidence for dramatic cooling of Caribbean surface waters as early as $4 \mathrm{Ma}$, a surface-water salinity increase probably accounts for the $\delta^{18} \mathrm{O}$ increase.

As discussed by Weyl (1968), the trade winds cause a net flux of water vapor from the Atlantic to the Pacific across Panama. This, in large part, causes North Atlantic surface waters to be on average $1 \%$ more saline than are North Pacific waters, with a maximum contrast of $2 \%_{0}$ on either side of the Panama Isthmus. Weyl believes that so long as there was an open tropical seaway between the Atlantic and the Pacific, mixing of surface waters prevented the salinity contrast we see today. Studies of the isotopic composition of seawater indicate that for the North Atlantic a surface-water salinity increase of $1 \%$ results in a $\delta^{18} \mathrm{O}$ increase of $0.60 \%$ (Craig and Gordon, 1965). The magnitude of the ${ }^{18} \mathrm{O}$ enrichment in the planktonic foraminifers ${ }^{18} \mathrm{O}(0.5$ to $1.0 \%)$ is equivalent to that predicted by a 1.0 to $2.0 \%$ surfacewater salinity increase in the North Atlantic. It is likely, 
then, that the early Pliocene oxygen isotope separation (Fig. 5) between Site 502 and 503 planktonic foraminifers reflects a change from well-mixed Atlantic-Pacific low-latitude surface waters to restricted communication by the emergent Panama Isthmus. Since the entire surface-North Atlantic is more saline than the surfaceNorth Pacific, the hypothesis of salinity change beginning approximately $4.2 \mathrm{Ma}$ is easily testable by further isotope studies of Pliocene-age deep-sea sequences elsewhere in the North Atlantic.

\section{CONCLUSIONS}

Analyses of stable isotopes were performed on monogeneric benthic and monospecific planktonic foraminifers from western Caribbean DSDP Site 502 and eastern equatorial Pacific Site 503. A late Miocene shift in carbon isotopes occurs at $6 \mathrm{Ma}$, and the inferred growth of permanent Northern Hemisphere continental ice cover is recorded at about $3.2 \mathrm{Ma}$. Comparing the results from each site highlights changes which may have resulted in part from the progressive uplift of the Central American land bridge and the development of the modern oceanic circulation system. These changes are:

1) At Atlantic Site 502, planktonic foraminiferal $\delta^{18} \mathrm{O}$ increases relative to the benthic $\delta^{18} \mathrm{O}$ values and relative to Pacific Site 502 planktonic foraminiferal $\delta^{18} \mathrm{O}$ at about $4.2 \mathrm{Ma}$. This change is probably related to restriction of tropical surface-water communication between the Atlantic and the Pacific by the emergent Panama Isthmus and to an increase in North Atlantic surface water salinity relative to the Pacific. The amount of the $\delta^{18} \mathrm{O}$ increase $(0.5$ to $1.0 \%)$ is close to that predicted from the modern surface-water salinity contrast between the Atlantic and Pacific oceans.

2) Deep-sea circulation became similar to the present-day pattern by $3 \mathrm{Ma}$, based on the geographic contrast of benthic foraminiferal $\delta^{13} \mathrm{C}$ between Sites 502 and 503. The development of the modern contrast occurred in two steps, each resulting in Caribbean benthic foraminifers becoming enriched in ${ }^{13} \mathrm{C}$, relative to $\mathrm{Pa}$ cific benthic forms. The first step occurred at the time of the late Miocene carbon shift (6 Ma) when the geographic contrast increased from about $0.60 \%$ to $0.75 \%$. This may have resulted from complete blocking of a previously limited deep-sea connection near Panama. At $3 \mathrm{Ma}$ the benthic $\delta^{13} \mathrm{C}$ contrast between the Atlantic and Pacific further increased from 0.75 to $1.00 \%$, the modern value at these locations, perhaps signalling increased production of nutrient-poor, oxygen-rich North Atlantic deep water.

3) The vertical contrast in carbon isotope ( $\delta$ planktonic minus $\delta$ benthic) has decreased at each site largely because of a greater decrease in planktonic than benthic $\delta^{13} \mathrm{C}$. The Pacific decrease may reflect an oceanwide chemistry change, such as a decline in the carbon-tophosphorus ratio, but the Atlantic decrease (which is twice as large) suggests additional local or basinwide effects. The significance of decreasing $\Delta \delta^{13} \mathrm{C}$ is unclear.

\section{ACKNOWLEDGMENTS}

Reviewed by W. B. Curry and D. F. Williams. I thank the Deep Sea Drilling Project for inviting me to join the scientific party aboard DSDP Leg 68 and the following people for their constructive com- ments on this manuscript: B. H. Corliss, W. B. Curry, D. W. Graham, T. S. Loutit, and D. F. Williams. I also thank M. L. Bender and J. P. Kennett for supporting me as a research associate in the CENOP program, funded through National Science Foundation Grant OCE7914594. Woods Hole Oceanographic Institution Contribution 4858.

\section{REFERENCES}

Belanger, P. E., Curry, W. B., and Matthews, R. K., 1981. Core-top evaluation of benthic foraminiferal isotopic ratios for paleooceanographic interpretations. Palaeogeog. Palaeoclim. Palaeoecol., 33:205-220.

Bender, M. L., and Keigwin, L. D., Jr., 1979. Speculations about the upper Miocene change in abyssal Pacific dissolved bicarbonate $\delta^{13}$ C. Earth Planet. Sci. Lett., 45:383-393.

Berger, W. H., 1970. Biogenous deep sea sediments: fractionation by deep-sea circulation. Geol. Soc. Am. Bull., 81:1385-1402.

Berger, W. H., Diester-Haass, L., and Killingley, J. S., 1978a. Upwelling off Northwest Africa: the Holocene decrease as seen in carbon isotopes and sedimentological indications. Oceanol. Acta, 1:1-7.

Berger, W. H., Killingley, J. S., and Vincent, E., 1978b. Stable isotopes in deep-sea carbonates: box core ERDC-92, west equatorial Pacific. Oceanol. Acta, 1:203-216.

Blanc, P.-L., Rabussier, D., Vergnaud-Grazzini, C., and Duplessy, J.C., 1980. North Atlantic Deep Water formed by the later middle Miocene. Nature, 283:553-555.

Broecker, W. S., 1974. Chemical Oceanography: New York (Harcourt Brace Jovanovich).

, in press. Glacial to interglacial changes in ocean chemistry. In Kraus, E. (Ed.), CIMAS Symposium Volume: Miami (University of Miami).

Craig, H., and Gordon, L. 1., 1965. Isotopic oceanography: deuterium and oxygen 18 variations in the ocean and marine atmosphere. In Schink, D. R., and Corless, J. T. (Eds.), Symposium on Marine Geochemistry: Narragansett Marine Laboratory Occasional Publication No. 3:277-374.

Duplessy, J.-C., Moyes, J., and Pujol, C., 1980. Deep water formation in the North Atlantic during the last ice age. Nature, 286:479.

Edgar, N. T., Saunders, J. B., et al., 1973. Init. Repts. DSDP, 15: Washington (U.S. Govt. Printing Office).

Graham, D. W., Corliss, B. H., Bender, M. L., and Keigwin, L. D., Jr., in press. Carbon and oxygen isotopic disequilibrium of Recent deep-sea benthic foraminifera. Mar. Micropaleontol.

Haq, B. U., Worsley, T. R., Burkle, L. H., Douglas, R. G., Keigwin, L. D., Jr., Opdyke, N. D., Savin, S. M., Sommer, M. A., Vincent, E., and Woodruff, F., 1980. Late Miocene marine carbon-isotopic shift and synchroneity of some phytoplanktonic biostratigraphic events. Geology, 8:427-431.

Hays, J. D., et al., 1972. Init. Repts. DSDP, 9: Washington (U.S. Govt. Printing Office).

Keigwin, L. D., Jr., 1978. Late Miocene carbon and oxygen isotope stratigraphy of east equatorial and north central Pacific Deep Sea Drilling Project (DSDP) sites. Abstracts with Programs: Toronto (Geological Society of American, Annual Meeting), p. 432.

1979. Late Cenozoic stable isotope stratigraphy and paleoceanography of DSDP sites from the east equatorial and central North Pacific Ocean. Earth Planet. Sci. Lett., 45:361-382.

1980. Paleoceanographic change in the Pacific at the Eocene-Oligocene boundary. Nature, 287:722-725.

Keigwin, L. D., Jr., Bender, M. L., and Kennett, J. P., 1979. Thermal structure of the deep Pacific Ocean in the early Pliocene. Science, 205:1386-1388.

Keigwin, L. D., Jr., and Shackleton, N. J., 1980. Uppermost Miocene carbon isotope stratigraphy of a piston core in the equatorial $\mathrm{Pa}$ cific. Nature, 284:613-614.

Keigwin, L. D., Jr., and Thunell, R. C., 1979. Middle Pliocene climatic change from faunal and oxygen isotopic trends: Western Mediterranean. Nature, 282:294-296.

Kroopnick, P., 1974. The dissolved $\mathrm{O}_{2}-\mathrm{CO}_{2}-{ }^{13} \mathrm{C}$ system in the eastern equatorial Pacific. Deep Sea Res., 21:211-227.

1980. The distribution of ${ }^{13} \mathrm{C}$ in the Atlantic Ocean. Earth Planet. Sci. Lett., 49:469-484.

Kroopnick, P., Weiss, R. F., and Craig, H., 1972. Total $\mathrm{CO}_{2},{ }^{13} \mathrm{C}$, and dissolved oxygen- ${ }^{18} \mathrm{O}$ at Geosecs II in the North Atlantic. Earth Planet. Sci. Lett., 16:103-110.

Lloyd, R. M., and Hsü, K. J., 1973. Preliminary isotopic investigations of samples from deep-sea drilling in the Mediterranean Sea. 
In Ryan, W. B. F., Hsü, K. J., et al., Init. Repts. DSDP, 13, Pt. 2, Washington (U.S. Govt. Printing Office), 783-787.

Loutit, T., and Kennett, J. P., 1979. Application of carbon isotope stratigraphy to late Miocene shallow marine sediments, New Zealand. Science, 204:1196-1199.

Moore, T. C., Pisias, N. G., and Keigwin, L. D., in preparation. Cenozoic variability of oxygen isotopes in benthic foraminifera. National Academy of Sciences Colloquium on pre-Quaternary Climates.

Prell, W. L., and Curry, W. B., 1981. Faunal and isotopic indices of monsoonal upwelling: Western Arabian Sea. Oceanol. Acta, 4: 91-98.

Shackleton, N. J., and Cita, M. B., 1979. Oxygen and carbon isotope stratigraphy of benthic foraminifera at Site 397: detailed history of climatic change during the late Neogene. In von Rad, U., Ryan, W. B. F., et al., Init. Repts. DSDP, 47, Pt. 1: Washington (U.S. Govt. Printing Office), 433-445.

Shackleton, N. J., and Kennett, J. P., 1975. Late Cenozoic oxygen and carbon isotopic changes at DSDP 284: Implications for glacial history of the Northern Hemisphere and Antarctica. In Kennett, J.
P., Houtz, R. E., et al., Init. Repts. DSDP, 29: Washington (U.S. Sovt. Printing Office), 801-807.

Shackleton, N. J., and Opdyke, N. D., 1977. Oxygen isotope and paleomagnetic evidence for early Northern Hemisphere glaciation. Nature, 270:216-219.

van Andel, Tj. H., 1975. Mesozoic/Cenozoic calcite compensation depth and the global distribution of calcareous sediment. Earth Planet. Sci. Lett., 26:187-194.

Vincent, E., Killingley, J. S., and Berger, W. H., 1980. The magnetic epoch 6 carbon shift: a change in the ocean's ${ }^{13} \mathrm{C} /{ }^{12} \mathrm{C}$ ratio 6.2 million years ago. Mar. Micropaleontol., 5:185.

Weyl, P. L., 1968. The role of the oceans in climatic change: a theory of the ice ages. Meteorological Monographs, 8:37-62.

Williams, D. F., Sommer, M. A., and Bender, M. L., 1977. Carbon isotopic compositions of Recent planktonic foraminifera of the Indian Ocean. Earth Planet. Sci. Lett., 36:391-403.

Woodruff, F., Savin, S. M., and Douglas, R. G., 1981. Miocene stable isotope record: A detailed deep Pacific Ocean study and its paleoclimatic implications. Science, 212:665-668. 